\title{
Solar feature tracking in both spatial and temporal domains
}

\author{
D. B. Jess ${ }^{1,2}$, M. Mathioudakis ${ }^{1}$, R. Erdélyi ${ }^{3}$, G. Verth $^{3}$, R. T. J. \\ McAteer $^{2}$ and F. P. Keenan ${ }^{1}$ \\ ${ }^{1}$ Astrophysics Research Centre, School of Mathematics and Physics, \\ Queen's University, Belfast, BT7 1NN, Northern Ireland, UK \\ email: djess01@qub.ac.uk \\ ${ }^{2}$ NASA Goddard Space Flight Center, Solar Physics Laboratory, \\ Code 671, Greenbelt, MD 20771, USA \\ ${ }^{3} \mathrm{SP}^{2} \mathrm{RC}$, Department of Applied Mathematics, The University of Sheffield, \\ Sheffield, S3 7RH, England, U.K.
}

\begin{abstract}
A new method for automated coronal loop tracking, in both spatial and temporal domains, is presented. The reliability of this technique was tested with TRACE $171 \AA$ observations. The application of this technique to a flare-induced kink-mode oscillation, revealed a $3500 \mathrm{~km}$ spatial periodicity which occur along the loop edge. We establish a reduction in oscillatory power, for these spatial periodicities, of $45 \%$ over a $322 \mathrm{~s}$ interval. We relate the reduction in oscillatory power to the physical damping of these loop-top oscillations.
\end{abstract}

Keywords. Sun: activity, Sun: atmospheric motions, Sun: corona, Sun: evolution, Sun: flares, Sun: oscillations

\section{Introduction}

Automated feature recognition and tracking has long been a goal for scientists. With the advent of higher sensitivity satellite-based telescopes and the resulting increase in readout rates, it is imperative to be able to analyse, at least to some minimal level, data "on the fly". Without this ability, the large data rates achievable make it extremely difficult to implement onboard trigger programmes (e.g. a flare trigger), which allow the telescope pointing to be redirected to an area of interest and modify the instrument observing mode, almost immediately. Furthermore, having a means of establishing interesting phenomena occurring on the Sun in real time allows instrument users to pick datasets of interest much more readily than in the past. Thus, many schemes have been setup to push this form of real-time data analysis forward, including the Heliophysics Knowledge Base programme, which will enable real-time detections of oscillatory phenomena occurring on the Sun.

Current orbiting solar satellites, in particular the Transition Region And Coronal Explorer, TRACE, have enabled the analysis of many oscillatory signatures originating within the outer atmosphere of the Sun. Wang \& Solanki (2004), Marsh \& Walsh (2006) and Schrijver et al. (2002) all report oscillatory phenomena occuring within coronal loop structures, thus demonstrating the need for automated, oscillation detection software. Previously, however, the majority of work on oscillating coronal loops has been focussed on the temporal domain (see latest review in Banerjee et al. 2007). In this work, we investigate the spatial evolution of a coronal loop structure, a method proposed first by Erdélyi \& Verth (2007); Verth et al. (2007), independent of its position during a flareinduced kink oscillation. From this we can understand the behaviour of the loop, in the 
spatial domain, and establish any phenomena which are flare induced, yet confined to the volume occupied by the loop. In $\S 2$ we describe the observations under investigation, while in $\S 3$ we discuss the methodologies used during the analysis of the data which allow us to accurately track the spatial evolution of coronal loops. A discussion of our results in the context of confined loop oscillations is given in $\S 4$, and finally, our concluding remarks are given in $\S 5$.

\section{Observations}

The data presented here are part of an observing sequence obtained on 1998 July 14, using the TRACE imaging satellite. The optical setup of TRACE allowed a $384^{\prime \prime} \times 384^{\prime \prime}$ region surrounding active region NOAA 8270 to be investigated with a spatial resolution of $1^{\prime \prime}\left(0.5^{\prime \prime}\right.$ per pixel). The filter selected for these observations was the $171 \AA$ filter, which has an inherent passband width of $6.4 \AA$, allowing plasma in the temperature range $0.2-$ 2.0 MK to be studied. The cadence of the TRACE instrument was not constant during the observing sequence, with the time between successive exposures varying between 66 and $82 \mathrm{~s}$. The observations employed in the present analysis consist of 88 successive TRACE images providing nearly two hours of continuous, uninterrupted data. During the observing sequence, a large M4.6 flare occurred in the immediate vicinity of the active region under investigation.

\section{Data Analysis}

\subsection{Initial Image Processing}

The TRACE data was retrieved directly from the Lockheed Martin Solar and Astrophysics Labs database and was subjected to common processing algorithms. The IDL routine TRACE_PREP.PRO was implemented calling appropriate keywords to remove cosmic-ray streaks, reduce readout noise and prepare the data in a user-friendly format. De-rotation of the TRACE images was also deemed necessary, since the observing sequence lasts approximately two hours, equating to a relatively large solar rotation (20" at disk centre). From this point, it was possible to use additional programmes to aid in the analysis of the data as described in detail below.

\subsection{Laplacian Image Sharpening}

The Laplacian operator is a second order method of enhancement and is particularly good at finding fine detail in an image. Any feature with a sharp discontinuity (such as noise) will unfortunately be enhanced by a Laplacian operator, however this method of image enhancement is often used when spatial resolutions are less than desirable. Thus, one application of the Laplacian operator is to restore fine detail to an image which has been smoothed, or pre-processed, to remove noise (eg using a median filter). The Laplacian operator is implemented as a convolution between an image and a kernel. The kernel can be constructed in various ways, but we use the 3-by-3 kernel implemented by Gonzalez \& Woods (1992).

In image convolution, the kernel is centered on each spatial pixel in turn, and the pixel value is replaced by the sum of the kernel mutipled by the image values. With the particular kernel implemented here, we are counting the contributions of the diagonal pixels as well as the orthogonal pixels in the filter operation. This is not always necessary or desirable, although it works well here, as will be demonstrated. Figure 1 shows the result of appling the convolution kernel to the TRACE image, as well as the final output after the fine-scale information has been added back into the original image. Notice 

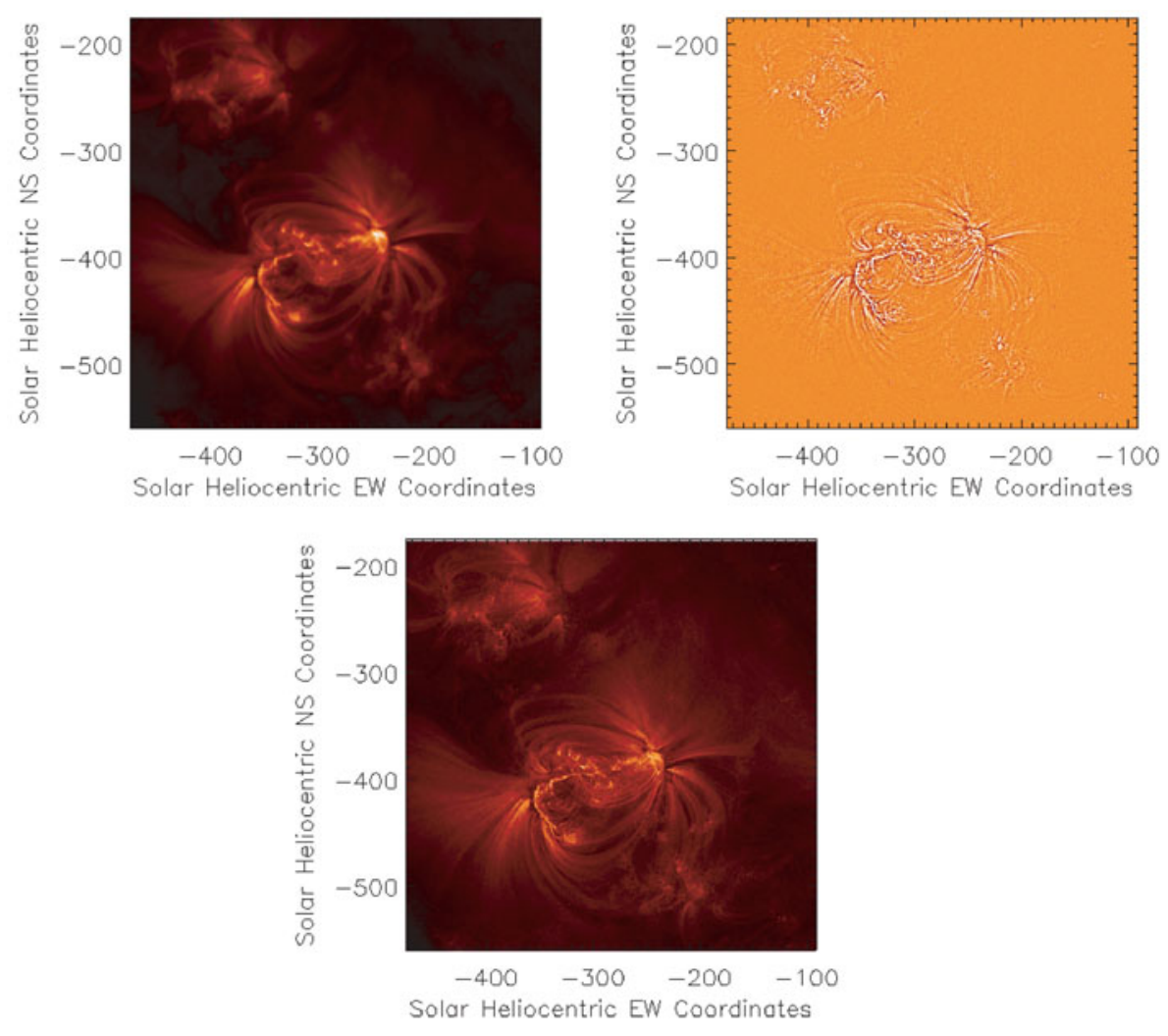

Figure 1. Complete TRACE field of view (top left) after undergoing initial processing using the TRACE_PREP.PRO IDL routine. The top right image shows the fine-scale structures detected using the Laplacian filter outlined in $\S 3.2$ and the bottom image reveals the sharpened TRACE field of view after the addition of fine-scale structures. Note how the clarity of loop structures is drastically increased.

the emphasis of fine structures when compared to the original, unfiltered image. For the purposes of the data under investigation here, all TRACE images were subjected to Laplacian image sharpening routines.

\subsection{Wavelet Modulous Maxima Edge Detection}

After sucessful completion of image sharpening, edge detection algorithms can be implemented. The sharpened images have increased fine-scale information and as such provide the necessary platform for establishing feature edges which may have been previously unresolvable. Immediately after the flare event, one particular coronal loop is seen to oscillate. This kink-mode oscillating loop has been investigated previously (see Aschwanden et al. 1999 for the initial investigation) in the temporal domain. Here, we will investigate the behaviour of the loop in the spatial domain. In order to track the spatial behaviour of the loop, a fully automated routine was devised to minimise errors introduced through human interaction with the data.

The first step involves intensity thresholding whereby features in the TRACE fieldof-view are contoured depending on their emissive flux. For the data presented here, a lower intensity threshold of the background median value plus 5 sigma is used. This value allowed us to contour TRACE coronal loop structures, yet leave out background quiet Sun. 

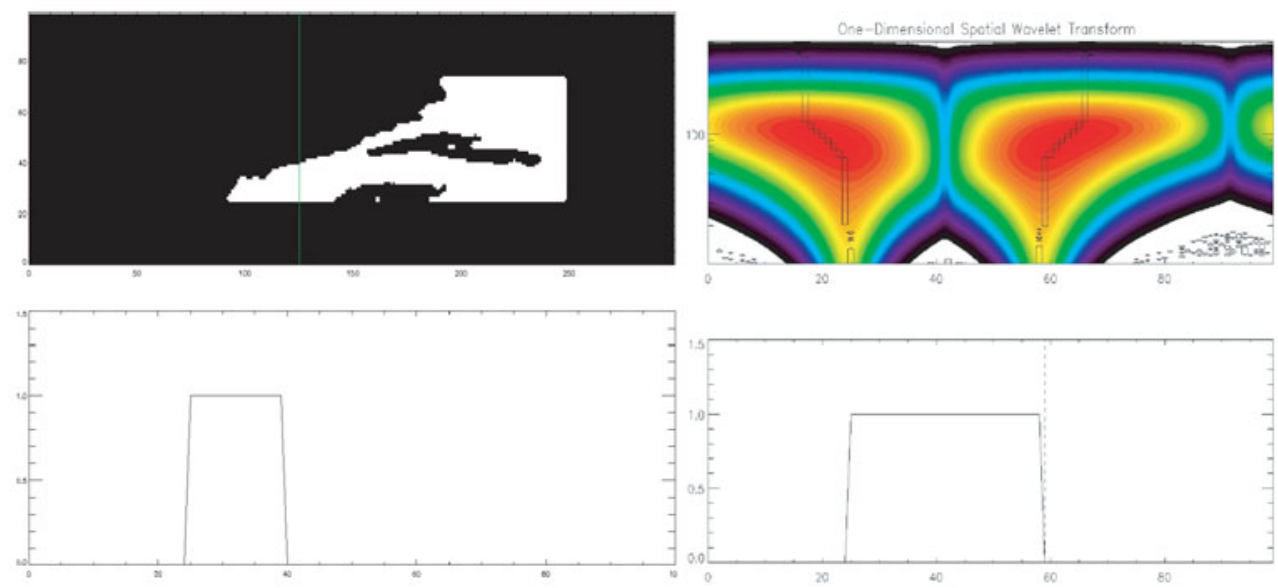

Figure 2. Upper-left panel shows the binary-formatted image obtained through intensity thresholding inserted into a padded, zeroed array which provides the best platform for WMM edge detection. The axis scales are pixel number with 1 pixel $=0.5^{\prime \prime}$. The vertical green line corresponds to $\mathrm{x}$ pixel element 125 with the resulting intensity plot for this $\mathrm{x}$ pixel shown in the bottom-left panel. The corresponding wavelet power spectrum (top right) generated along the vertical spatial direction of the TRACE field of view for horizontal pixel number 125 . This panel also shows regions of maximum power being traced down to the intersect point on the x-axis. The intensity plot (same as lower left) is again plotted (bottom right) with a dashed line indicating the position of intersection of the maximum wavelet power traces with the x-axis. Notice how the $\mathrm{x}$-axis intersect position of maximum wavelet power corresponds exactly to the reduction of intensity in the bottom panel. This reduction in intensity is analagous with the upper edge of the TRACE coronal loop showing the accuracy of this method when detecting feature edges.

To emphasize feature edges and remove shallow intensity gradients, a binary format for feature mapping is used. All pixels which lay above the lower intensity threshold defined above are assigned a value of ' 1 '. Those pixels which lay below the threshold were assigned a value of ' 0 ' as seen in Figure 2. This binary format provides an absolute intensity cut-off thus providing a definite feature edge which can be tracked, both spatially and temporally. The binary image is padded using a zeroed array from which feature edge tracking can commence.

Tracking of this feature edge is performed using a Wavelet Modulous Maxima (WMM) technique (Muzy et al. 1993, McAteer et al. 2007). From the padded binary array created above, a horizontal pixel is chosen. Figure 2 shows the selection of horizontal pixel 125 , as indicated by the vertical green line, as well as the corresponding intensity plot along this vertical line. A wavelet transform is produced for this intensity plot, demonstrated in the right-hand panel of Figure 2, utilizing a Mexican Hat wavelet. A Mexican Hat wavelet is very useful for detecting sharp intensity gradients which are present due to the binary format implemented above. An absolute wavelet power spectrum is plotted as a function of vertical pixel element in Figure 2. Additionally, maximum wavelet power features are traced down to the $\mathrm{x}$-axis of the power spectrum and reveal the locations of maximum intensity gradients. Figure 2 re-plots the intensity in the vertical direction at horizontal pixel 125 and it can be seen that where the maximum wavelet power meets the x-axis also corresponds to the edges of the TRACE coronal loop under investigation.

Due to the positioning of the loop with respect to the neighbouring active region, better loop contrast is achieved at the Northern edge of the loop where the brightness of the loop overlies fainter, less intense, quiet Sun. Contrarily, the Southern edge of the loop is difficult to separate from other loop structures, due to the densely packed nature of 
such features closer to the centre of active regions. Since in this instance it is desirable to track the upper edge of the coronal loop, we disregard the first maximum wavelet power location and record the position where the second maximum wavelet power location intersects with the x-axis. This pin-points the exact location of the Northerly edge of the coronal loop in question. This process is repeated over the entire $\mathrm{E} / \mathrm{W}$ direction, and for all frames in the observing sequence, with the subsequent position of the Northern edge of the TRACE coronal loop recorded. From the resulting loop edge positions, it is possible to analyse both temporal and spatial variations.

\section{Results and Discussion}

It is our main goal to investigate the spatial behaviour of the oscillating coronal loop, as mentioned in $\S 3.3$. However, to verify that the presented loop-tracking routine is functioning properly, derived results in the temporal domain are compared to previous findings. Examining the temporal behaviour of the detected coronal-loop edge, a periodicity of approximately $300 \mathrm{~s}$ exists which is consistent with the findings of Aschwanden et al. (1999). This result implies that the loop detection and tracking algorithms, developed here, are functioning accurately, as temporal variations of loop position are consistent with previously published results.

From examination of the TRACE time series, it appears that the oscillating coronal loop passes through an equilibrium position at times of 0,280 and $602 \mathrm{~s}$, with the overall shape of the loop similar at the corresponding TRACE frames. If indeed the shape of the loop is identical at times of 0,280 and $602 \mathrm{~s}$, then a subtraction of any two of these loop shapes should provide a resulting image equal to zero. However, upon subtraction of the loop shape at $0 \mathrm{~s}$ from that at $280 \mathrm{~s}$, a spatial periodicity is revealed. Running this resulting loop shape through a one-dimensional spatial wavelet transform establishes an oscillatory period along the Northern loop edge of 10 pixels (Fig. 3). A number of strict criteria implemented on the data allowed us to insure that oscillatory signatures correspond to real periodicities. These criteria have been described in detail in previous papers (see Banerjee et al. 2001, Jess et al. 2007, McAteer et al. 2004). With the TRACE plate scale equal to $0.5^{\prime \prime}$ per pixel, the detected 10 pixel periodicity corresponds to an absolute spatial distance of approximately $3500 \mathrm{~km}$ per complete oscillation (more than three complete cycles detected).

To see if this periodicity is visible at the following equilibrium position (602 s), we subtract the loop shape at $0 \mathrm{~s}$ from that at $602 \mathrm{~s}$, and pass through our one-dimensional spatial wavelet transform. The resulting evidence indicates that the 10 pixel spatial periodicity is indeed still present, albeit with a reduction in oscillatory power. We interpret the reduction in oscillatory power as the physical signature of damping of the loop-top oscillations. A closer inspection reveals that the oscillatory power has dropped by $45 \%$ during the $322 \mathrm{~s}$ interval, remaining consistent with other aspects of coronal-loop oscillations, which are seen to be heavily damped (Aschwanden et al. 2003, Ruderman 2005, Terradas et al. 2006, Dymova \& Ruderman 2007).

To investigate a possible phase relationship (Athay \& White 1978), we create a crosswavelet power spectrum between the two detected loop-top oscillations, and inspect the resulting spatial-phase diagram (Fig. 4). This figure reveals a clear phase shift, over many complete wavelengths, in the order of $0^{\circ}$ to $-50^{\circ}$. Of course, due to the long kink-oscillation period $(\approx 300 \mathrm{~s})$, and the relatively poor, and irregular, cadence of the TRACE instrument, we are only able to evaluate kink-equilibrium spatial periodicities at two temporal locations. At these two locations, the coronal loop undergoing the kink oscillation has returned to it's equilibrium state - i.e. matching the shape and position of 

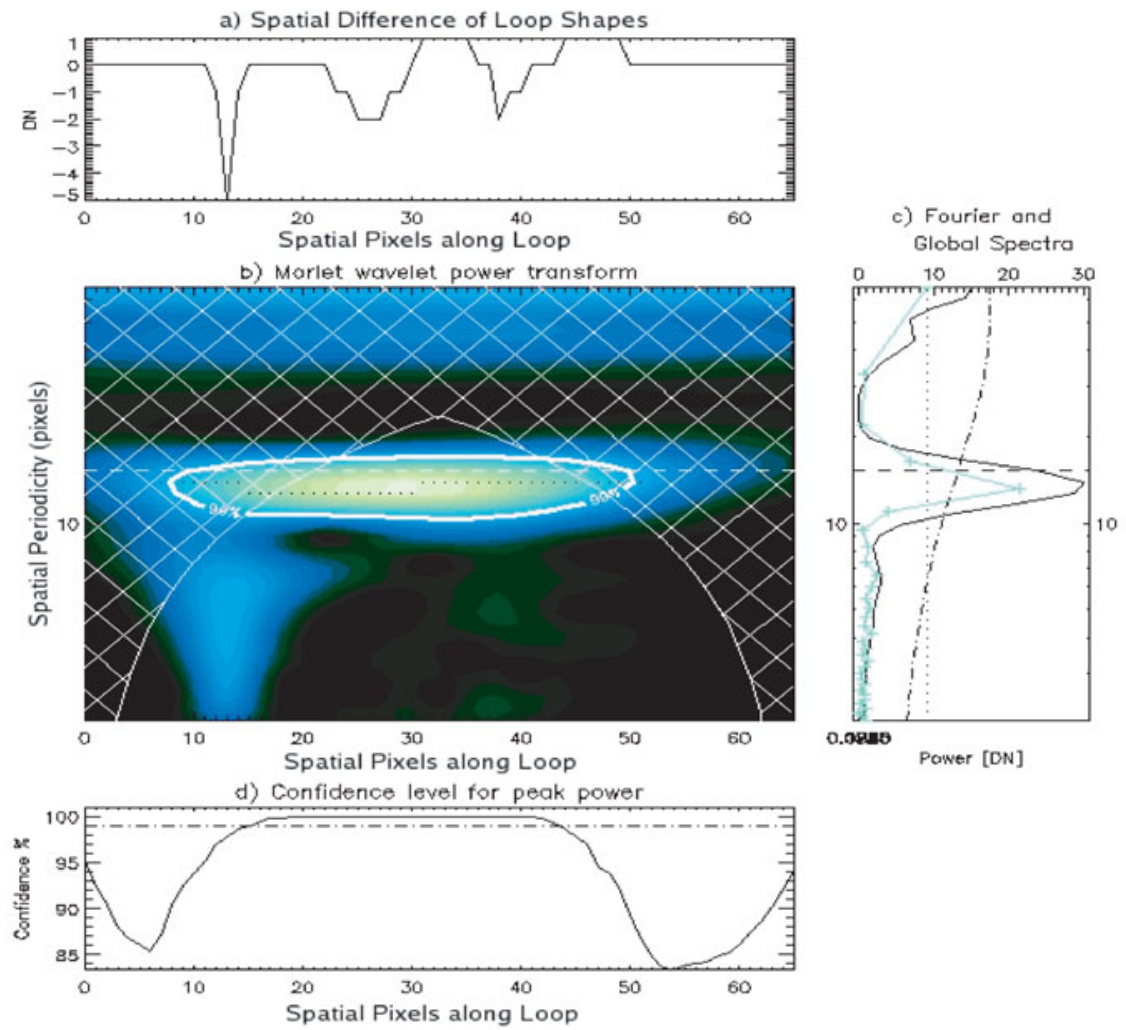

Figure 3. Detection of a 10 pixel spatial oscillation. The resulting Northern loop-edge outline created by the subtraction of the loop edge at $0 \mathrm{~s}$ from the loop edge at $280 \mathrm{~s}$ after the flare event is shown in a). The one-dimensional spatial wavelet power transform along with locations where detected power is at, or above, the $99 \%$ confidence level are contained within the contours in b). Plot c) shows the summation of the wavelet power transform over time (full line) and the Fast Fourier power spectrum (crosses) over time, plotted as a function of period. Both methods have detected a well pronounced 10 pixel spatial oscillation. The global wavelet (dotted line) and Fourier (dashed dotted line) 95\% significance levels are also plotted. The cone of influence (COI), cross-hatched area in the plot, defines an area in the wavelet diagram where edge effects become important and as such any frequencies outside the COI are disregarded. Periods above the horizontal line (dotted) fall within the COI. The probability levels, which are related to the percentage confidence attributed to the peak power at every time step in the wavelet transform, are plotted in d).

the loop prior to the flare-induced kink oscillation. Between these two equilibrium states, the loop in question has underwent one complete kink-oscillation cycle. If this loop-top oscillation is a propagating wave using the confines of the coronal loop as a waveguide, then it must exhibit a velocity matching the Alfvén speed. Assuming an Alfvén speed of $\approx 1000 \mathrm{~km} / \mathrm{s}$ (Nakariakov \& Verwichte 2005), and using the time interval (322 s) between successive equilibrium positions, we can derive a traversed distance for the loop-top wave equating to $\approx 0.32 \mathrm{Mm}$ (equivalent to 88.9 periods). Subtracting complete periods from this value $(88.9-88)$, we are left with 0.9 , which corresponds to a phase of $324^{\circ}$. This is comparable to the $0^{\circ}$ to $-50^{\circ}$ phase relation shown in Fig. 4.

In order to validate our results, we have set up a number of further rigorous tests. We have implemented the same loop-edge detection and tracking processes on additional 

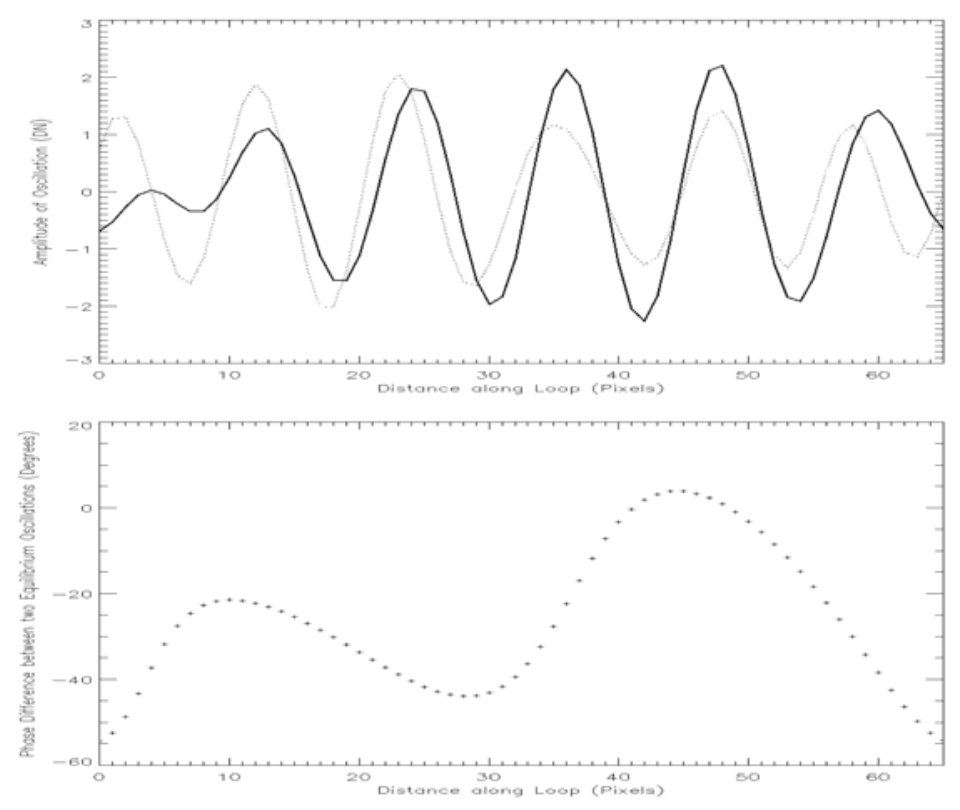

Figure 4. The top panel shows the oscillation amplitude as a function of position along the loop for both loop-top oscillations. The solid line relates to the spatial periodicity detected between times of 0 and $280 \mathrm{~s}$, while the dotted line relates to the spatial periodicity detected between times of 0 and $602 \mathrm{~s}$. From this plot, it is clear to see a phase shift between the two loop-top oscillations. The bottom panel shows the phase-difference results of a cross-wavelet spectrum of the two detected spatial oscillations. We can clearly see a phase shift, on the order of $0^{\circ}$ to $-50^{\circ}$, over the duration of 6 complete cycles.

coronal loops away from the region under investigation, yet within the same TRACE field of view. Since these loops are not connected with the flare event, and therefore not seen to oscillate, no spatial periodicities along the loop would be expected when two loop outlines are subtracted from one and other. Subtracting the control loop outline at $0 \mathrm{~s}$ from that at $280 \mathrm{~s}$ after the flare, to remain consistent with the work above, and running through a one-dimensional spatial wavelet transform reveals no periodicities. Furthermore, we tested for spatial periodicities, on the loop seen to oscillate, both prior to, and long after, the flare event. These tests found no oscillatory phenomena in either instance, indicating that the spatial periodicities detected are flare induced.

\section{Concluding Remarks}

A new method for the detection, and subsequent tracking, of coronal loop structures is presented. This method also provides the ability to simultaneously search for longitudinal and transverse oscillations in real-time. This is particularly useful for future space-bourne solar telescopes, where the large achievable data rates will make real-time data analysis paramount. Utilizing our method for automated coronal loop analysis, we have detected spatial periodicities, on the order of $3500 \mathrm{~km}$ per cycle, occurring on a coronal loop immediately after being buffeted by a neighbouring M4.6 flare. We detect the damping of these loop-top oscillations, with a $45 \%$ reduction in oscillatory power over a $322 \mathrm{~s}$ interval. We also detect a wrapped spatial phase shift of $0^{\circ}$ to $-50^{\circ}$, related to the time interval of $322 \mathrm{~s}$ between successive kink-oscillation equilibrium positions, for the travelling loop-top oscillations. 


\section{Acknowledgements}

DBJ wishes to thank the Department for Employment and Learning and NASA's Goddard Space Flight Center for a studentship. RE is grateful for an IAU travel grant, acknowledges M. Kéray for patient encouragement, and, is also grateful to NSF, Hungary (OTKA, Ref. No. K67746). Wavelet software was provided by C. Torrence and G. Compo.

\section{References}

Aschwanden, M. J., Fletcher, L., Schrijver, C. J., \& Alexander, D., 1999, ApJ, 520, 880

Aschwanden, M. J., Nightingale, R. W., Andries, J., Goossens, M., \& Van Doorsselaere, T., 2003, ApJ, 598, 1375

Athay, R. G., \& White, O. R., 1978, ApJ, 226, 1135

Banerjee, D., Erdélyi, R., Oliver, R. \& O'Shea, E., 2007, Sol. Phys, 246, 3

Banerjee, D., O'Shea, E., Doyle, J. G., \& Goossens, M., 2001, A\&\&A, 371, 1137

Dymova, M. V., \& Ruderman, M. S., 2007, A\&A, 463, 759

Erdélyi, R. \& Verth, G., 2007, A\& A, 642, 743

Gonzalez, R., \& Woods, R., 1992, Digital Image Processing, Addison Wesley, 414

Jess, D. B., Andić, A., Mathioudakis, M., Bloomfield, D. S., \& Keenan, F. P., 2007, A\&̋A, 473, 943

McAteer, R. T. J., Gallagher, P. T., Bloomfield, D.S., Williams, D. R., Mathioudakis, M., \& Keenan, F.P., 2004, ApJ, 602, 436

McAteer, R. T. J., Young, C. A., Ireland, J., \& Gallagher, P. T., 2007, ApJ, 662, 691

McEwan, M. P., \& De Moortel, I., 2006, A\&AA, 448, 763

Marsh, M. S., \& Walsh, R. W., 2006, ApJ, 643, 540

Muzy, J. F., Bacry, E., \& Arneodo, A., 1993, Phys. Rev. A, E-47, 875

Nakariakov, V. M., \& Verwichte, E., 2005, LRSP, 3

Ruderman, M. S., 2005, Proc. Dynamic Sun : Challenges for Theory and Observations, ESA-SP 600,96

Schrijver, C. J., Aschwanden, M. J., \& Title, A. M., 2002, Solar Phys., 206, 69

Sheeley, N. R., Wang, Y. M., Hawley, S. H., Brueckner, G. E., Dere, K. P., et. al., 1997, ApJ, 484,472

Terradas, J., Oliver, R., \& Ballester, J. L., 2006, ApJ, 650, 91

Verth, G., van Doorsselaere, T., Erdélyi, R. \& Goossens, M., 2007, A\&AA, 475, 341

Wang, T. J., \& Solanki, S. K., 2004, A\&A, 421, 33 